\title{
A coupled level set projection method applied to ink jet simulation
}

\author{
JIUN-DER Yu ${ }^{\dagger}$ \\ Epson Research and Development, Inc., \\ 3145 Porter Drive, Suite 104, Palo Alto, CA 94304, USA \\ SHINRI SAKA ${ }^{\ddagger}$ \\ Seiko Epson Corporation, TP Development Department, \\ 80 Harashinden, Hirooka, Shiojiri-shi, Nagano-ken, 399-0785, Japan \\ AND \\ J. A. SETHian ${ }^{\S}$ \\ Department of Mathematics, University of California at Berkeley, \\ Berkeley, CA 94720, USA
}

[Received 18 October 2002 and in revised form 7 May 2003]

\begin{abstract}
A finite difference level set projection method on rectangular grid is developed for piezoelectric ink jet simulation. The model is based on the Navier-Stokes equations for incompressible twophase flows in the presence of surface tension and density jump across the interface separating ink and air, coupled to an electric circuit model which describes the driving mechanism behind the process, and a macroscopic contact model which describes the air-ink-wall dynamics. We simulate the axisymmetric flow using a combination of second-order projection methods to solve the fluid equations and level set methods to track the air/ink interface. The numerical method can be used to analyze the motion of the interface, breakoff and formation of satellites, and effect of nozzle geometry on droplet size and motion. We focus on close comparison of our numerical ink jet simulation with experimental data.
\end{abstract}

\section{Introduction}

The goal of this work is to develop a numerical simulation tool for fluid flow phenomena associated with ink jet printers. The underlying algorithms should be able to accurately capture two-phase flow through an axisymmetric nozzle, handle complicated topological change of ink droplets, conserve mass to a good approximation, and couple to external models which simulate the ink cartridge, supply channel, pressure chamber, and piezoelectric actuator.

In Figure 1. we show the typical structure of an ink jet nozzle; the actual geometry is axisymmetric and is not drawn to scale. Ink is stored in a bath reservoir (cartridge), and driven through the nozzle in response to a pressure jump at the lower boundary. The dynamics of incompressible flow through the nozzle, coupled to surface tension effects along the ink-air interface

\footnotetext{
† The corresponding author. Email: yu.jiunder@erd.epson.com

Email: Sakai.Shinri@exc.epson.co.jp

${ }^{\S}$ This author was supported in part by the Applied Mathematical Sciences subprogram of the Office of Energy Research, U.S. Department of Energy, under Contract Number DE-AC03-76SF00098, and the Division of Mathematical Sciences of the National Science Foundation. Email: sethian@math.berkeley.edu
} 
and boundary conditions along the wall, act to determine the shape of the interface as it moves. A negative pressure at the lower boundary induces a backflow which causes a bubble to pinch off, separate, and move through the domain.

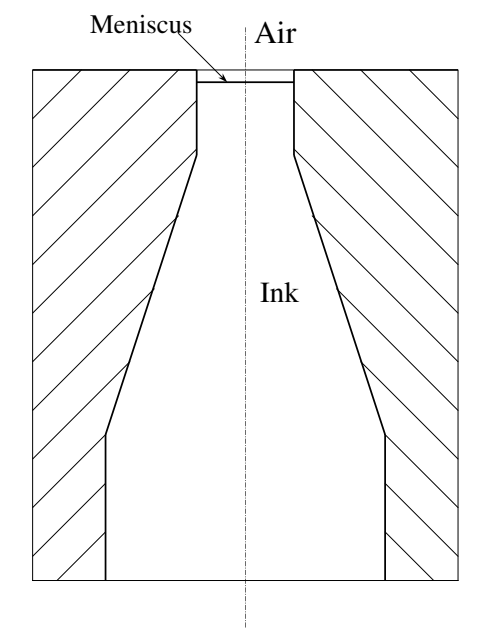

FIG. 1. The cross section view of an ink jet nozzle.

The model is based on the Navier-Stokes equations for two-phase immiscible incompressible flows in the presence of surface tension and density jump across the interface separating ink and air, coupled to an electric circuit model which describes the driving mechanism behind the process, and a macroscopic slipping contact line model which describes the air-ink-wall dynamics. The fluid interface between the air and the ink is treated as an infinitely thin immiscible boundary, separating regions of differing but constant densities and viscosities. The flow is axisymmetric, and for boundary conditions on solid walls we assume that both the normal and tangential components of the fluid velocity vanish; this is amended through a contact model which describes the motion of the triple point which describes the air-fluid-wall interaction. We simulate axisymmetric flow using a combination of second-order projection methods to solve the fluid equations and level set methods to track the air/ink boundary. The model is used to analyze the motion of the boundary, breakoff and formation of satellites, and the effect of nozzle geometry on ink ejection size and motion.

Several different numerical simulations of this process have been performed in recent years; see, for example, Aleinov et al. [1] and Sou et al. [20]. There are many numerical techniques to solve the Navier-Stokes equations, including finite difference techniques, finite element techniques, and spectral techniques, as well as methods to track the fluid interface, including marker particle techniques and volume-of-fluid techniques. We employ second-order finite difference projection methods to solve the fluid flow equations and level set methods to capture the motion of the fluid interface.

Projection methods for incompressible Navier-Stokes equations were originally developed by Chorin [9]. They work by first solving the Navier-Stokes equations to predict the intermediate velocity fields and then projecting the velocity onto the space of divergence-free fields to enforce the incompressibility condition. This method was improved by Bell, Colella, and Glaz [4] in the late 1980's with the use of a second-order Godunov-type scheme for the nonlinear convection term. 
The implementation of the projection step was then made easier through the introduction of a finiteelement approximate projection by Almgren et al. [3]. The extension of these methods to two-fluid problems adds extra complexity to the "projection" algorithm in several ways. First, the region occupied by any one of the two fluids keeps changing. Second, surface tension at the interface contributes an extra term in the governing equations. Third, since the density and viscosity are not constant, the viscosity term is no longer a product of the Laplacian of the velocity and the viscosity.

Level set methods, introduced in Osher and Sethian [13], rely in part on the theory of curve and surface evolution given in Sethian [16, 17] and on the link between front propagation and hyperbolic conservation laws discussed in Sethian [18]. They recast interface motion as a timedependent Eulerian initial value partial differential equation, and rely on viscosity solutions to the appropriate differential equations to update the position of the front, using an interface velocity that is derived from the relevant physics both on and off the interface.

For details about projection methods and their coupling to level set methods, see Almgren et al. [3, 2], Bell et al. [4], Bell and Marcus [5], Chang et al. [7], Chorin [9], Puckett et al. [14], Sussman and Smereka [22], Sussman et al. [23, 21], and Zhu and Sethian [26].

In this paper, we carefully examine the effects of various contact models and equivalent circuit driving models on the computed results, and closely compare our results with experimental data. We provide relevant equations of motion, details of the underlying algorithm, and detailed numerical tests of robustness and accuracy, followed by simulations of the ink jet process with comparison to experiment. The paper is meant to provide a clear description of the underlying equations, algorithms and programming details. There is considerable previous work on secondorder projection methods coupled to level set methods for fluid flow, and we refer the interested readers to these works; they have served as a foundation for some of what is discussed in this paper.

\section{Level set formulation}

\subsection{Equations of motion}

Both of the fluids are governed by the incompressible Navier-Stokes equations, i.e.

$$
\rho_{i} \frac{D \boldsymbol{u}_{i}}{D t}=-\nabla p_{i}+\nabla \cdot\left(2 \mu_{i} \mathcal{D}\right), \quad \nabla \cdot \boldsymbol{u}_{i}=0, \quad i=1,2 .
$$

In the above equations,

$$
\mathcal{D}=\frac{1}{2}\left[\nabla \boldsymbol{u}+(\nabla \boldsymbol{u})^{T}\right], \quad \boldsymbol{u}=u e_{1}+v e_{2},
$$

are the rate of deformation tensor and the fluid velocity, respectively, $\frac{D}{D t}=\frac{\partial}{\partial t}+(\boldsymbol{u} \cdot \nabla)$ is the Lagrangian time derivative, $p$ the pressure, $\rho$ the density, and $\mu$ the dynamic viscosity. The subscript $i=1,2$ is used to denote the variable or constant in fluid \#1 (ink) or fluid \#2 (air). The boundary conditions at the interface of the two phases are the continuity of the velocity and the jump condition

$$
\left(2 \mu_{1} \mathcal{D}-2 \mu_{2} \mathcal{D}\right) \cdot \boldsymbol{n}=\left(p_{1}-p_{2}+\sigma \kappa\right) \boldsymbol{n},
$$

where $\boldsymbol{n}$ is the unit normal to the interface drawn from fluid \#2 to fluid \#1 and $\kappa$ is the curvature of the interface. Because the size of typical ink jet print heads is small, the gravity term is not important and is omitted. We note that the inclusion of a gravity term does not change any part of the numerical scheme described in the next section. 
We use the level set method to trace the interface (see Osher and Sethian [13]). The interface is the zero level of the level set function $\phi$,

$$
\phi(x, y, t) \begin{cases}<0 & \text { if }(x, y) \in \text { fluid \#2 (air), } \\ =0 & \text { if }(x, y) \in \text { interface, } \\ >0 & \text { if }(x, y) \in \text { fluid \#1 (ink). }\end{cases}
$$

Here, the level set function $\phi$ is initialized as the signed distance to the interface. The unit normal on the interface can be expressed in terms of $\phi$ :

$$
\boldsymbol{n}=\left.\frac{\nabla \phi}{|\nabla \phi|}\right|_{\phi=0}, \quad \kappa=\left.\nabla \cdot\left(\frac{\nabla \phi}{|\nabla \phi|}\right)\right|_{\phi=0} .
$$

Let

$$
\boldsymbol{u}= \begin{cases}\boldsymbol{u}_{1}, & \phi>0, \\ \boldsymbol{u}_{2}, & \phi<0 .\end{cases}
$$

The governing equations for the two-phase flow and the boundary condition at the interface can be re-written as

$$
\begin{gathered}
\nabla \cdot \boldsymbol{u}=0, \\
\rho(\phi) \frac{D \boldsymbol{u}}{D t}=-\nabla p+\nabla \cdot(2 \mu(\phi) \mathcal{D})-\sigma \kappa(\phi) \delta(\phi) \nabla \phi,
\end{gathered}
$$

where $\delta$ is the Dirac delta function. We note that the Navier-Stokes equations for two-phase flows 2.8) have been written in similar form and used by Unverdi and Tryggvason [24]. The fact that the surface tension can be written as a body force concentrated at the interface has been used by Unverdi and Tryggvason as well as others. The form [2.8 is due to Chang et al. [7].

To make the governing equations dimensionless, we choose the following definitions:

$$
\begin{aligned}
& x=L x^{\prime}, \quad y=L y^{\prime}, \quad \boldsymbol{u}=U \boldsymbol{u}^{\prime}, \quad t=\frac{L}{U} t^{\prime}, \\
& p=\rho_{1} U^{2} p^{\prime}, \quad \rho=\rho_{1} \rho^{\prime}, \quad \mu=\mu_{1} \mu^{\prime},
\end{aligned}
$$

where the primed quantities are dimensionless and $L, U, \rho_{1}, \mu_{1}$ are respectively the characteristic length, characteristic velocity, density of fluid \#1, and dynamic viscosity of fluid \#1.

Substituting the above into equations 2.7 and 2.8 , and dropping the primes, we have

$$
\begin{gathered}
\nabla \cdot \boldsymbol{u}=0, \\
\frac{D \boldsymbol{u}}{D t}=-\frac{1}{\rho(\phi)} \nabla p+\frac{1}{\rho(\phi) \operatorname{Re}} \nabla \cdot(2 \mu(\phi) \mathcal{D})-\frac{1}{\rho(\phi) \mathrm{We}} \kappa(\phi) \delta(\phi) \nabla \phi,
\end{gathered}
$$

where the density ratio, viscosity ratio, Reynolds number, and Weber number are defined by

$$
\begin{aligned}
\rho(\phi) & = \begin{cases}1 & \text { if } \phi \geqslant 0, \\
\rho_{2} / \rho_{1} & \text { if } \phi<0,\end{cases} \\
\mu(\phi) & = \begin{cases}1 & \text { if } \phi \geqslant 0, \\
\mu_{2} / \mu_{1} & \text { if } \phi<0,\end{cases} \\
\operatorname{Re} & =\frac{\rho_{1} U L}{\mu_{1}}, \\
\mathrm{We} & =\frac{\rho_{1} U^{2} L}{\sigma} .
\end{aligned}
$$


Since the interface moves with the fluid, the evolution of the level set is governed by

$$
\frac{\partial \phi}{\partial t}+\boldsymbol{u} \cdot \nabla \phi=0
$$

We choose this form because the interface moves advectively.

Since equations 2.5, 2.10, and 2.11] are expressed in terms of the vector notation, they assume the same form in Cartesian coordinates and axisymmetric coordinates. In axisymmetric coordinates $(r, z)$, the curvature can be expanded as

$$
\kappa(\phi)=\nabla \cdot\left(\frac{\nabla \phi}{|\nabla \phi|}\right)=\frac{\phi_{r r} \phi_{z}^{2}-2 \phi_{r z} \phi_{r} \phi_{z}+\phi_{z z} \phi_{r}^{2}+\phi_{r}\left(\phi_{r}^{2}+\phi_{z}^{2}\right) / r}{\left(\phi_{r}^{2}+\phi_{z}^{2}\right)^{3 / 2}},
$$

where the subscripts $r$ and $z$ denote the partial derivatives, i.e.

$$
\phi_{r} \equiv \frac{\partial \phi}{\partial r}, \quad \phi_{z} \equiv \frac{\partial \phi}{\partial z}, \quad \phi_{r z} \equiv \frac{\partial^{2} \phi}{\partial r \partial z}, \quad \text { and so on. }
$$

By including appropriate definitions of the derivatives, the viscosity term becomes

$$
\begin{aligned}
\nabla \cdot[2 \mu(\phi) \mathcal{D}]= & \left\{\frac{1}{r} \frac{\partial}{\partial r}\left(2 \mu(\phi) r \frac{\partial u}{\partial r}\right)-2 \mu(\phi) \frac{u}{r^{2}}+\frac{\partial}{\partial z}\left[\mu(\phi)\left(\frac{\partial v}{\partial r}+\frac{\partial u}{\partial z}\right)\right]\right\} e_{r} \\
& +\left\{\frac{1}{r} \frac{\partial}{\partial r}\left[\mu(\phi) r\left(\frac{\partial v}{\partial r}+\frac{\partial u}{\partial z}\right)\right]+\frac{\partial}{\partial z}\left(2 \mu(\phi) \frac{\partial v}{\partial z}\right)\right\} e_{z} .
\end{aligned}
$$

\subsection{Boundary conditions}

On solid walls, we assume that both the normal and tangential components of the velocity vanish (this must be amended at the triple point, which we discuss below). At both inflow and outflow, our formulation allows us to specify either velocity or pressure boundary conditions. Time-dependent inflow conditions are provided by an equivalent circuit model which mimics the charge-driven mechanism which forces ink from the bath into the nozzle.

\subsection{Contact models}

At the triple point, where air and ink meet at the solid wall, several choices are available for the boundary conditions.

- We can choose to enforce the no-slip condition. In this case, the triple point will remain fixed, since the fluid does not move tangential to the walls, and produce a very long fluid bubble which remains attached to the wall.

- We can choose to enforce some sort of condition which restricts the critical angle that is allowed, this means that the triple point will move. To do so without connecting this movement to the fluid velocity violates conservation of mass.

- A third option is to relax the no-slip condition in the neighborhood of the triple point once the critical angle is exceeded; the idea here is to allow the local flow at the triple point to quickly accelerate whenever the critical angle is exceeded (see, for example, Bertozzi [6]). 


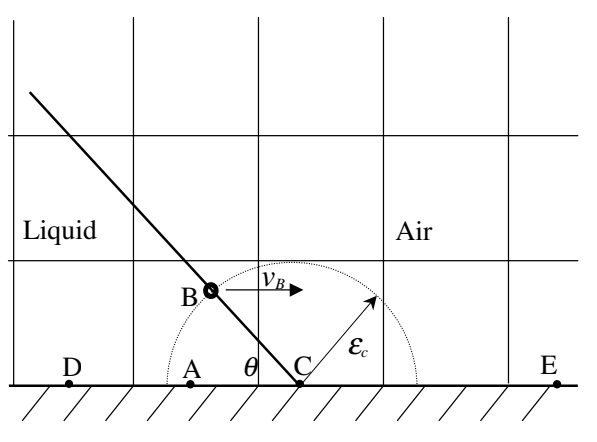

Solid

FIG. 2. An ink-air interface and the contact point.

In this paper, we adopted the following slipping contact line model. Consider the air-liquid interface and the contact line shown in Fig. 2. We first define the contact angle $\theta$ as the angle made by the air-liquid interface and the solid, measured from the side of the liquid by approaching the contact line (i.e. the triple point) as close as possible. The advancing critical contact angle $\theta_{a}$ and receding critical contact angle $\theta_{r}$ are the maximum and minimum contact angles for the triple point to stay. In most situations $\theta_{r}<\theta_{a}$. But the receding critical angle may be very close or even equal to the advancing critical angle. Actual values of $\theta_{a}$ and $\theta_{r}$ depend on both the fluids and the solid surface. The velocity $v_{B}$ is the tangential velocity of the air-liquid interface at point $B$, which is located on the interface and is $\xi_{C}$ from the contact point. For Epson's dye-based ink and print head nozzle wall, $\theta_{a}$ and $\theta_{r}$ are about $70^{\circ}$ and $20^{\circ}$. We used $\xi_{C}=0.5 \mu \mathrm{m}$ in all the numerical computations presented in this work.

We use the following criteria to decide whether to let the triple point move:

- The triple point is allowed to move toward the air side if $\theta \geqslant \theta_{a}$ and $v_{B}>0$.

- The triple point is allowed to move toward the liquid side if $\theta \leqslant \theta_{r}$ and $v_{B}<0$.

- The triple point should not move otherwise.

We note that according to this model, the triple point is not allowed to move toward the ink (air) side if $\theta>\theta_{a}\left(\theta<\theta_{r}\right)$.

If the triple point is not allowed to move, the boundary condition at the solid wall is the no-slip condition. If the triple point is allowed to move, the no-slip condition in a close vicinity of the triple point is switched to the free slip condition. In this work, we chose to set two cells at each side of the contact point to free slip should the triple point be allowed to move. For example, the cells containing $A, C$, and $D$ are given the free slip boundary condition while the cell containing $E$ is given the no-slip condition (since most part of its solid wall is more than two cells from the contact point).

We note that, applying the above contact model, the slipping velocity of the contact point is always decided by the flow instead of by anything artificial.

\subsection{Equivalent circuit}

In a piezo ink jet print head, the formation of the ink droplet is controlled by a piece of piezoelectric PZT (lead zirconate titanate) actuator. Driven by the input voltage, the PZT pushes and then pulls 
the ink. To numerically simulate an ink jet, one needs to prescribe a velocity or pressure at the inflow to the nozzle. However, only the input voltage to the PZT is known.

An equivalent circuit model can be used to model the problem. The ink flow rate and pressure are first taken as dependent variables. Each component of the ink jet print head, such as the nozzle, pressure chamber, vibration plate, PZT actuator, and ink cartridge, is expressed in terms of the compliance and acoustic resistance. These acoustics elements are finally transferred to their equivalent inductance, capacitance, and electric resistance to form an equivalent circuit. By solving the equivalent circuit and the flow equations in turn, one simulates a real ink jet.

The particular equivalent circuit we use is not described here; readers are referred to Sakai [15] for an introduction to print head structures and equivalent circuits. A typical driving voltage pattern and a typical inflow pressure are as shown in Figures 3 and 4 . The driving voltage is such that the

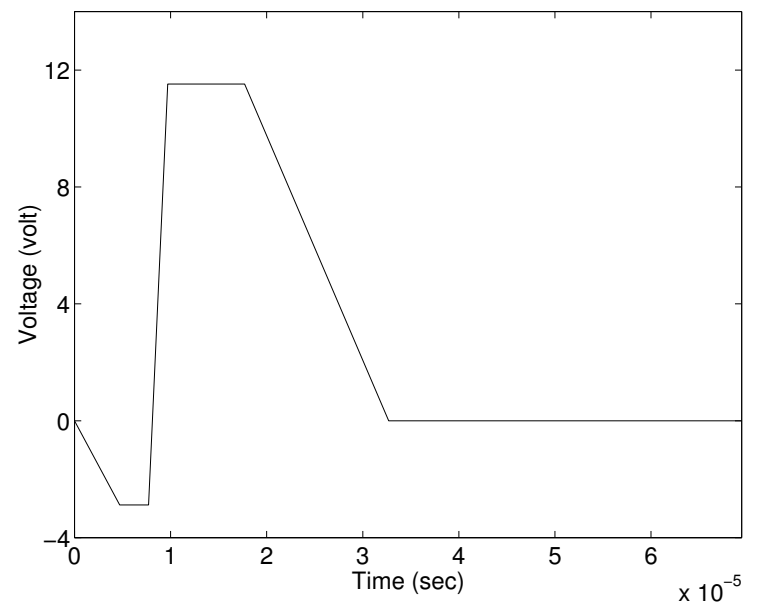

FIG. 3. A typical ink jet driving voltage (one cycle).

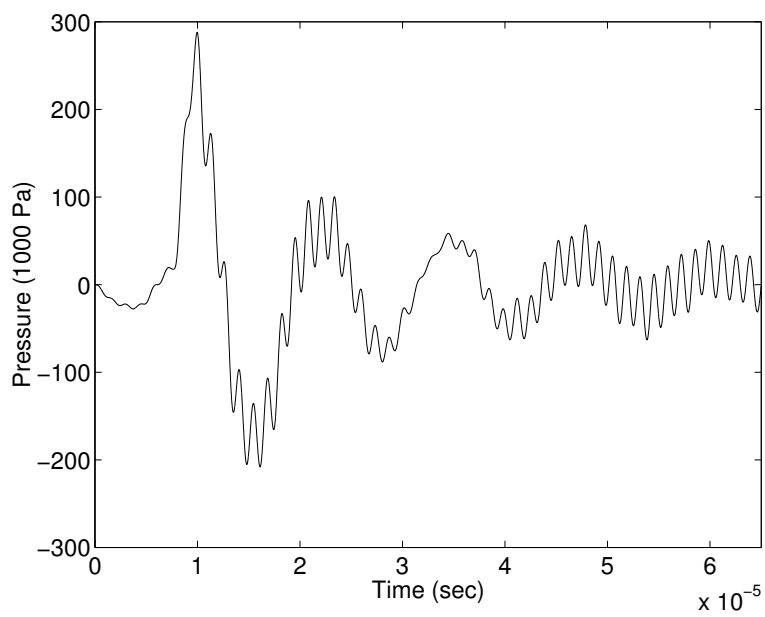

FIG. 4. A typical inflow pressure (one cycle). 
ink is first pulled back, pushed and fired, and then pulled back to get ready for the next ejection. The inflow pressure shown in Figure 4 reflects the reaction of a typical nozzle-ink channel-PZT-cartridge system to the applied voltage. The pressure pattern contains several high frequency signals. The biggest one is basically the fundamental natural frequency of the system, which is five to six times higher than the driving voltage frequency in this case. The small oscillations are probably related to the natural frequencies of the components in the system.

\section{Numerical algorithms on rectangular grids}

In the paper, the superscript $n$ (or $n+1)$ denotes the time step, i.e.

$$
\boldsymbol{u}^{n}=\boldsymbol{u}(t=n \Delta t)
$$

and so on. Given quantities $\boldsymbol{u}^{n}, p^{n}, \phi^{n}$, the purpose is to obtain $\boldsymbol{u}^{n+1}, p^{n+1}, \phi^{n+1}$ which satisfy the condition of incompressibility 2.10. The explicit algorithm we describe will be first-order accurate in time and second-order accurate in space.

\subsection{Temporal discretization}

The boundary condition on the nozzle wall stems from the proposed contact model. The inflow pressure at $t^{n+1}$ is given by the equivalent circuit.

3.1.1 Level set update. The level set is updated by

$$
\phi^{n+1}=\phi^{n}-\Delta t[\boldsymbol{u} \cdot \nabla \phi]^{n+1 / 2} .
$$

The time-centered advection term $[u \cdot \nabla \phi]^{n+1 / 2}$ is evaluated using an explicit predictor-corrector scheme that requires only the available data at $t^{n}$; more detail is given in Section 3.2 Once $\phi^{n+1}$ is obtained, we compute $\phi^{n+1 / 2}$ by using the update rule

$$
\phi^{n+1 / 2}=\frac{1}{2}\left(\phi^{n}+\phi^{n+1}\right) \text {. }
$$

3.1.2 Explicit algorithm for Navier-Stokes equations. In Puckett et al. [14] and Sussman et al. [21], the temporal discretization is explicit for the advection term and semi-implicit for the viscosity term. In our work, an explicit temporal discretization is applied to the viscosity term to limit the compute time spent on the inversion of the viscosity term: this explicit temporal discretization is given by

$$
\begin{aligned}
\frac{\boldsymbol{u}^{n+1}-\boldsymbol{u}^{n}}{\Delta t}+[(\boldsymbol{u} \cdot \nabla) \boldsymbol{u}]^{n+1 / 2}= & -\frac{1}{\rho\left(\phi^{n+1 / 2}\right)} \nabla p^{n+1}+\frac{1}{\rho\left(\phi^{n+1 / 2}\right) \operatorname{Re}} \nabla \cdot\left[2 \mu\left(\phi^{n+1 / 2}\right) \mathcal{D}^{n}\right] \\
& -\frac{1}{\rho\left(\phi^{n+1 / 2}\right) \mathrm{We}}[\kappa(\phi) \delta(\phi) \nabla \phi]^{n+1 / 2}
\end{aligned}
$$

If we define

$$
\begin{array}{r}
\boldsymbol{u}^{*}=\boldsymbol{u}^{n}+\Delta t\left\{-[(\boldsymbol{u} \cdot \nabla) \boldsymbol{u}]^{n+1 / 2}+\frac{1}{\rho\left(\phi^{n+1 / 2}\right) \operatorname{Re}} \nabla \cdot\left[2 \mu\left(\phi^{n+1 / 2}\right) \mathcal{D}^{n}\right]\right. \\
\left.-\frac{1}{\rho\left(\phi^{n+1 / 2}\right) \mathrm{We}}[\kappa(\phi) \delta(\phi) \nabla \phi]^{n+1 / 2}\right\},
\end{array}
$$


the time-discretized Navier-Stokes equations can be written as

$$
\boldsymbol{u}^{n+1}=\boldsymbol{u}^{*}-\frac{\Delta t}{\rho\left(\phi^{n+1 / 2}\right)} \nabla p^{n+1} .
$$

We apply a second-order explicit Godunov scheme for the advection term and the central difference for the viscosity term in 3.1 .2 . They will be explained later. It is clear that the determination of $\boldsymbol{u}^{*}$ needs only values at time step $n$.

3.1.3 Projection for $\boldsymbol{u}^{n+1}$. To satisfy the incompressibility condition for time step $n+1$, we apply the divergence operator on both sides of 3.6. Since $\nabla \cdot \boldsymbol{u}^{n+1}=0$, we have

$$
\nabla \cdot \boldsymbol{u}^{*}=\nabla \cdot\left(\frac{\Delta t}{\rho\left(\phi^{n+1 / 2}\right)} \nabla p^{n+1}\right)
$$

The projection equation (3.7) is elliptic. It reduces to a Poisson equation if the density ratio $\rho\left(\phi^{n+1 / 2}\right)$ is a constant. After the pressure $p^{n+1}$ is solved from equation 3.7), the velocity field $\boldsymbol{u}^{n+1}$ can be obtained by 3.6.

To simplify the implementation for arbitrary geometries, we use a finite element projection of the form

$$
\int_{\Omega} \boldsymbol{u}^{*} \cdot \nabla \psi \mathrm{d} \boldsymbol{x}=\int_{\Omega} \frac{\Delta t}{\rho\left(\phi^{n+1 / 2}\right)} \nabla p^{n+1} \cdot \nabla \psi \mathrm{d} \boldsymbol{x}+\int_{\Gamma_{1}} \psi \boldsymbol{u}^{B C} \cdot \boldsymbol{n} \mathrm{d} S,
$$

where $\psi$ is the finite element weighting function, $\Gamma_{1}$ denotes all the boundary with inflow or outflow, and $\boldsymbol{u}^{B C}$ is the given boundary velocity. It is easy to see by the divergence theorem that the implied boundary condition at $\Gamma_{1}$ is

$$
\frac{\Delta t}{\rho\left(\phi^{n+1 / 2}\right)} \frac{\partial p^{n+1}}{\partial n}=\left(\boldsymbol{u}^{*}-\boldsymbol{u}^{B C}\right) \cdot \boldsymbol{n} .
$$

The use of the finite element projection 3.8 in an otherwise finite difference scheme was first proposed by Almgren et al. [3], where the finite element projection was called an "approximate projection." This idea was then applied to variable density flows by Puckett et al. [14]. The choice of the weighting function, as well as the approximation for the pressure and velocity, is flexible. In our implementation, the weighting function and the pressure are chosen to be piecewise bilinear, and the velocity be piecewise constant.

3.1.4 Re-initialization of the level set. To correctly capture the interface and accurately calculate the surface tension, the level set function should remain a signed distance function to the interface as the calculation unfolds. However, if we update the level set by 2.10), it will not remain as such. Instead, we periodically stop the simulation and recreate a new level set function $\phi$ which is the signed distance function, i.e. $|\nabla \phi|=1$, without changing the zero level set of the original level set function. Details about re-initialization times and criteria can be found in Sethian [19].

The need to do so in level set calculations was first recognized by Chopp [8]. Re-initialization was an integral part of the level set fluid calculations in Sussman et al. [23]. A direct and simple method for re-initialization is to first find the interface (the zero level set) using a standard contour 
plotting algorithm and then recalculate the signed distance from each cell to the interface. Another simple re-initialization choice is to solve the crossing time problem

$$
\phi_{t^{\prime}}+F|\nabla \phi|=0
$$

where $F$ is a given normal velocity. We note that we have used $t^{\prime}$ in the equation to emphasize that it is a pseudo time variable and the equation is solved solely for the purpose of re-initialization. With $F=1$, we flow the interface forwards and backwards in time and calculate the time $t^{\prime}$ at which the level set function changes sign for each cell. The crossing times (both positive and negative) are equal to the signed distances.

We have tried both of the two simple methods in our code and do not notice any differences in simulation results. Readers are referred to Sethian [19] for an introduction to other re-initialization techniques.

\subsection{Approximation of the advection term}

The algorithm for the advection terms is based on the unsplit Godunov method introduced by Colella [11].

(a)

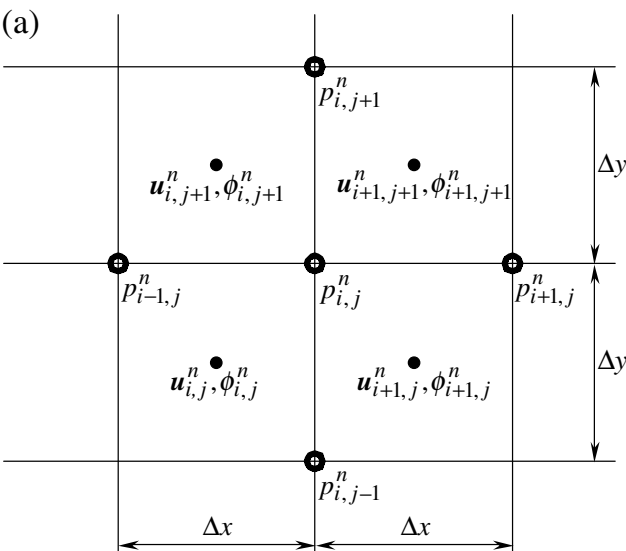

(b)

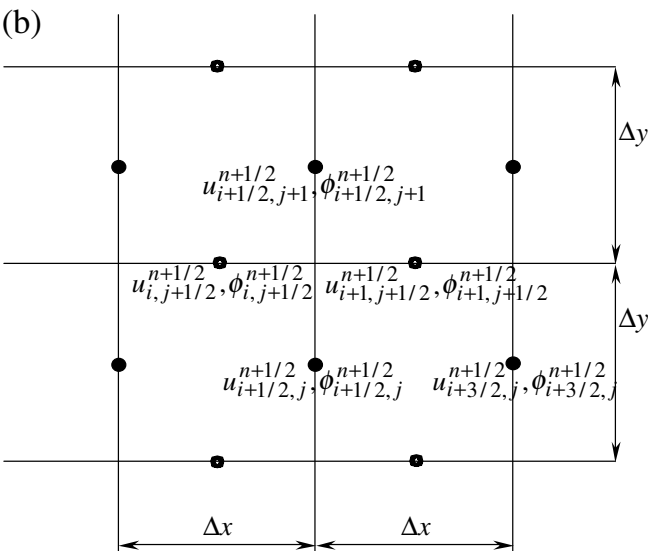

FIG. 5. Location of variables: (a) discrete velocity field, pressure and level set; (b) intermediate velocity field and level set.

Referring to Figures 5 (a) and 5 (b), the velocity components $\boldsymbol{u}_{i, j}^{n}$ and the level set function values $\phi_{i, j}^{n}$ are located at cell centers, and the pressure $p_{i, j}^{n}$ is located at grid points. The time-centered edge velocities and level set function values (also called the "predictors"), such as $\boldsymbol{u}_{i+1 / 2, j}^{n+1 / 2}, \phi_{i+1 / 2, j}^{n+1 / 2}$, and so on, are located at the middle point of each edge. To evaluate the advection terms, we use

$$
\begin{aligned}
{[(\boldsymbol{u} \cdot \nabla) \boldsymbol{u}]_{i, j}^{n+1 / 2}=} & \frac{u_{i+1 / 2, j}^{n+1 / 2}+u_{i-1 / 2, j}^{n+1 / 2}}{2} \frac{\boldsymbol{u}_{i+1 / 2, j}^{n+1 / 2}-\boldsymbol{u}_{i-1 / 2, j}^{n+1 / 2}}{\Delta r} \\
& +\frac{v_{i, j+1 / 2}^{n+1 / 2}+v_{i, j-1 / 2}^{n+1 / 2}}{2} \frac{\boldsymbol{u}_{i, j+1 / 2}^{n+1 / 2}-\boldsymbol{u}_{i, j-1 / 2}^{n+1 / 2}}{\Delta z},
\end{aligned}
$$




$$
\begin{aligned}
{[(\boldsymbol{u} \cdot \nabla) \phi]_{i, j}^{n+1 / 2}=} & \frac{u_{i+1 / 2, j}^{n+1 / 2}+u_{i-1 / 2, j}^{n+1 / 2}}{2} \frac{\phi_{i+1 / 2, j}^{n+1 / 2}-\phi_{i-1 / 2, j}^{n+1 / 2}}{\Delta r} \\
& +\frac{v_{i, j+1 / 2}^{n+1 / 2}+v_{i, j-1 / 2}^{n+1 / 2}}{2} \frac{\phi_{i, j+1 / 2}^{n+1 / 2}-\phi_{i, j-1 / 2}^{n+1 / 2}}{\Delta z} .
\end{aligned}
$$

In the above equations, the edge velocities and edge level sets are obtained by using a Taylor expansion in space and time. The time derivatives $\boldsymbol{u}_{t}^{n}$ and $\phi_{t}^{n}$ are given by the Navier-Stokes equations and the level set convection equation. We extrapolate from both sides of the edge and then apply the Godunov type upwinding to decide which extrapolation to use. Here we explain in detail how to obtain $\boldsymbol{u}_{i+1 / 2, j}^{n+1 / 2}$; the other time-centered edge values may be derived in a similar manner.

To begin, extrapolate from the left:

$$
\begin{aligned}
\boldsymbol{u}_{i+1 / 2, j}^{n+1 / 2, L} & =\boldsymbol{u}_{i, j}^{n}+\frac{\Delta r}{2} \boldsymbol{u}_{r, i, j}^{n}+\frac{\Delta t}{2} \boldsymbol{u}_{t, i, j}^{n} \\
& =\boldsymbol{u}_{i, j}^{n}+\frac{1}{2}\left(\Delta r-\Delta t u_{i, j}^{n}\right) \boldsymbol{u}_{r, i, j}^{n}-\frac{\Delta t}{2}\left(\widehat{v \boldsymbol{u}}_{z}\right)_{i, j}+\frac{\Delta t}{2} \boldsymbol{F}_{i, j}^{n},
\end{aligned}
$$

where

$$
\boldsymbol{F}_{i, j}^{n}=\left\{-\frac{1}{\rho(\phi)} \nabla p+\frac{1}{\rho(\phi) \mathrm{Re}} \nabla \cdot(2 \mu(\phi) \mathcal{D})-\frac{1}{\rho(\phi) \mathrm{We}} \kappa(\phi) \delta(\phi) \nabla \phi\right\}_{i, j}^{n} .
$$

We then extrapolate from the right:

$$
\begin{aligned}
\boldsymbol{u}_{i+1 / 2, j}^{n+1 / 2, R} & =\boldsymbol{u}_{i+1, j}^{n}-\frac{\Delta r}{2} \boldsymbol{u}_{r, i+1, j}^{n}+\frac{\Delta t}{2} \boldsymbol{u}_{t, i+1, j}^{n} \\
& =\boldsymbol{u}_{i+1, j}^{n}-\frac{1}{2}\left(\Delta r+\Delta t u_{i+1, j}^{n}\right) \boldsymbol{u}_{r, i+1, j}^{n}-\frac{\Delta t}{2}\left(\widehat{v \boldsymbol{u}}_{z}\right)_{i+1, j}+\frac{\Delta t}{2} \boldsymbol{F}_{i+1, j}^{n} .
\end{aligned}
$$

We use the monotonicity-limited central difference scheme (Colella [10]) for the evaluation of the normal slopes, which is $\boldsymbol{u}_{r, i, j}^{n}$ in this case. The limiting is done on each component of the velocity at $t^{n}$ separately. The transverse derivative term $\left(\widehat{v}_{z}\right)_{i, j}$ is evaluated by first extrapolating $\boldsymbol{u}$ to the transverse faces from the cell center, using normal derivatives only, and then applying the Godunov type upwinding. The detail will be given later in Subsections 3.2.1 and 3.2.2

The next step is the Godunov upwinding. We choose $\boldsymbol{u}_{i+1 / 2, j}^{n+1 / 2, L}$ or $\boldsymbol{u}_{i+1 / 2, j}^{n+1 / 2, R}$ according to

$$
\begin{aligned}
& u_{i+1 / 2, j}^{n+1 / 2}= \begin{cases}u_{i+1 / 2, j}^{n+1 / 2, L} & \text { if } u_{i+1 / 2, j}^{n+1 / 2, L}>0 \text { and } u_{i+1 / 2, j}^{n+1 / 2, L}+u_{i+1 / 2, j}^{n+1 / 2, R}>0, \\
u_{i+1 / 2, j}^{n+1 / 2, R} & \text { if } u_{i+1 / 2, j}^{n+1 / 2, R}<0 \text { and } u_{i+1 / 2, j}^{n+1 / 2, L}+u_{i+1 / 2, j}^{n+1 / 2, R}<0, \\
0 & \text { otherwise, }\end{cases} \\
& v_{i+1 / 2, j}^{n+1 / 2}\left(\text { or } \phi_{i+1 / 2, j}^{n+1 / 2}\right)= \begin{cases}v_{i+1 / 2, j}^{n+1 / 2, L}\left(\text { or } \phi_{i+1 / 2, j}^{n+1 / 2, L}\right) & \text { if } u_{i+1 / 2, j}^{n+1 / 2}>0, \\
v_{i+1 / 2, j}^{n+1 / 2, R}\left(\text { or } \phi_{i+1 / 2, j}^{n+1 / 2, R}\right) & \text { if } u_{i+1 / 2, j}^{n+1 / 2}<0, \\
\frac{v_{i+1 / 2, j}^{n+1 / 2, L}+v_{i+1 / 2, j}^{n+1 / 2, R}}{2}\left(\text { or } \frac{\phi_{i+1 / 2, j}^{n+1 / 2, L}+\phi_{i+1 / 2, j}^{n+1 / 2, R}}{2}\right) & \text { if } u_{i+1 / 2, j}^{n+1 / 2}=0 .\end{cases}
\end{aligned}
$$


These intermediate edge velocities are, in general, not divergence-free. We need to use an intermediate marker-and-cell (MAC) projection to make all the normal edge velocities divergencefree. Suppose $q$ is a function which is smooth enough and $\boldsymbol{u}^{e}$ the edge velocities obtained as in (3.13)-3.17). We want

$$
\boldsymbol{u}^{e}-\frac{1}{\rho\left(\phi^{n}\right)} \nabla q
$$

to be divergence-free. By taking the divergence of (3.18), we have

$$
\nabla \cdot\left(\frac{1}{\rho\left(\phi^{n}\right)} \nabla q\right)=\nabla \cdot \boldsymbol{u}^{e} .
$$

Since we apply the MAC projection for edge velocities, we let $q$ be cell-centered. Multiplying 3.19] by $r$ for axisymmetric coordinates, we have

$$
\frac{\partial}{\partial r}\left(\frac{r}{\rho(\phi)} \frac{\partial q}{\partial r}\right)+\frac{\partial}{\partial z}\left(\frac{r}{\rho(\phi)} \frac{\partial q}{\partial z}\right)=\frac{\partial}{\partial r}\left(r u^{e}\right)+\frac{\partial}{\partial z}\left(r v^{e}\right),
$$

where we have omitted the superscript $n$ for the level set. Hence, the discretized equation is given by

$$
\begin{gathered}
\frac{1}{\Delta r^{2}}\left[\frac{r_{i+1 / 2, j}}{(\rho(\phi))_{i+1 / 2, j}}\left(q_{i+1, j}-q_{i, j}\right)-\frac{r_{i-1 / 2, j}}{(\rho(\phi))_{i-1 / 2, j}}\left(q_{i, j}-q_{i-1, j}\right)\right] \\
+\frac{r_{i, j}}{\Delta z^{2}}\left[\frac{1}{(\rho(\phi))_{i, j+1 / 2}}\left(q_{i, j+1}-q_{i, j}\right)-\frac{1}{(\rho(\phi))_{i, j-1 / 2}}\left(q_{i, j}-q_{i, j-1}\right)\right] \\
=\frac{r_{i+1 / 2, j} u_{i+1 / 2, j}^{n+1 / 2}-r_{i-1 / 2, j} u_{i-1 / 2, j}^{n+1 / 2}}{\Delta r}+\frac{r_{i, j}\left(v_{i, j+1 / 2}^{n+1 / 2}-v_{i, j-1 / 2}^{n+1 / 2}\right)}{\Delta z} .
\end{gathered}
$$

The boundary condition for the intermediate projection should be compatible with the physical boundary conditions. On solid walls, $\boldsymbol{u} \cdot \boldsymbol{n}=0$, which is already used in the construction of the edge velocity $\boldsymbol{u}^{e}$; thus, we have a homogeneous Neumann boundary condition on $q$, namely $\partial q / \partial n=0$. At the inflow or outflow, if a velocity is prescribed, it is again a homogeneous Neumann boundary condition on $q$, since the given velocity at $t^{n+1 / 2}$ has been included in obtaining $\boldsymbol{u}^{e}$. If a pressure is given, the corresponding condition on $q$ is

$$
q=\frac{\Delta t}{2} p^{\text {boundary }},
$$

where the factor 2 in the denominator appears because the edge velocity is time-centered.

After $q_{i, j}$ is solved, we replace all the normal edge velocities by

$$
\begin{aligned}
& u_{i+1 / 2, j}^{n+1 / 2} \leftarrow u_{i+1 / 2, j}^{n+1 / 2}-\frac{1}{(\rho(\phi))_{i+1 / 2, j}} \frac{q_{i+1, j}-q_{i, j}}{\Delta r}, \\
& v_{i, j+1 / 2}^{n+1 / 2} \leftarrow v_{i, j+1 / 2}^{n+1 / 2}-\frac{1}{(\rho(\phi))_{i, j+1 / 2}} \frac{q_{i, j+1}-q_{i, j}}{\Delta z} .
\end{aligned}
$$


3.2.1 Evaluation of the normal derivatives. The normal derivatives in (3.13) and $(3.15)$ are evaluated using the fourth-order monotonicity-limited central difference scheme by Colella [10]. For uniform rectangular grid, we show how to calculate $u_{r, i, j}$. We begin with the following difference operator definitions:

$$
\begin{aligned}
D_{r}^{c}(u)_{i, j} & =\left(u_{i+1, j}-u_{i-1, j}\right) / 2, \\
D_{r}^{+}(u)_{i, j} & =\left(u_{i+1, j}-u_{i, j}\right), \\
D_{r}^{-}(u)_{i, j} & =\left(u_{i, j}-u_{i-1, j}\right) .
\end{aligned}
$$

These operators are then used to define the limiting slope

$$
\delta_{\lim }(u)_{i, j}= \begin{cases}\min \left(2\left|D_{r}^{-}(u)_{i, j}\right|, 2\left|D_{r}^{+}(u)_{i, j}\right|\right) & \text { if }\left(D_{r}^{-}(u)_{i, j}\right)\left(D_{r}^{+}(u)_{i, j}\right)>0, \\ 0 & \text { otherwise, }\end{cases}
$$

and the second-order limited slope

$$
\delta_{f}(u)_{i, j}=\min \left(\left|D_{r}^{c}(u)_{i, j}\right|, \delta_{\lim }(u)_{i, j}\right) \times \operatorname{sign}\left(D_{r}^{c}(u)_{i, j}\right) .
$$

The fourth-order limited slope is given by

$$
\delta_{r}^{4}(u)_{i, j}=\min \left(\left|\frac{4 D_{r}^{c}(u)_{i, j}}{3}-\frac{\delta_{f}(u)_{i+1, j}+\delta_{f}(u)_{i-1, j}}{6}\right|, \delta_{\lim }(u)_{i, j}\right) \times \operatorname{sign}\left(D_{r}^{c}(u)_{i, j}\right),
$$

and the fourth-order monotonicity-limited normal derivative is simply

$$
u_{r, i, j}=\delta_{r}^{4}(u)_{i, j} / \Delta r .
$$

3.2.2 Evaluation of the tangential derivatives. In Taylor's extrapolations 3.13 and 3.15, the normal derivatives are computed using the monotonicity-limited central difference scheme. However, to ensure stability, the tangential derivative should be computed using some sort of upwind schemes. Here we illustrate one possible approach, namely

$$
\left(\widehat{v \boldsymbol{u}}_{z}\right)_{i, j}=\frac{\hat{v}_{i, j+1 / 2}^{\mathrm{adv}}+\hat{v}_{i, j-1 / 2}^{\mathrm{adv}}}{2} \frac{\hat{\boldsymbol{u}}_{i, j+1 / 2}-\hat{\boldsymbol{u}}_{i, j-1 / 2}}{\Delta z} .
$$

In the above equation, $\hat{\boldsymbol{u}}_{i, j+1 / 2}$ and $\hat{v}_{i, j+1 / 2}^{\mathrm{adv}}$ are again derived from Taylor's extrapolation from the top and bottom of the edge. Neglecting pressure, viscosity, and surface tension terms as well as the tangential derivatives, this yields

$$
\begin{aligned}
& \hat{\boldsymbol{u}}_{i, j+1 / 2}^{B}=\boldsymbol{u}_{i, j}^{n}+\frac{1}{2}\left(\Delta z-\Delta t v_{i, j}^{n}\right) \boldsymbol{u}_{z, i, j}^{n}, \\
& \hat{\boldsymbol{u}}_{i, j+1 / 2}^{T}=\boldsymbol{u}_{i, j+1}^{n}-\frac{1}{2}\left(\Delta z+\Delta t v_{i, j+1}^{n}\right) \boldsymbol{u}_{z, i, j+1}^{n} .
\end{aligned}
$$

The derivatives at the right hand side are again computed using the monotonicity-limited central difference described in Subsection 3.2.1

All that remains is to perform the upwind evaluation to obtain $\hat{\boldsymbol{u}}_{i, j+1 / 2}^{n}$ and $\hat{v}_{i, j+1 / 2}^{\mathrm{adv}}$ :

$$
\hat{v}_{i, j+1 / 2}^{\text {adv }}= \begin{cases}\hat{v}_{i, j+1 / 2}^{B} & \text { if } \hat{v}_{i, j+1 / 2}^{B}>0 \text { and } \hat{v}_{i, j+1 / 2}^{B}+\hat{v}_{i, j+1 / 2}^{T}>0, \\ \hat{v}_{i, j+1 / 2}^{T} & \text { if } \hat{v}_{i, j+1 / 2}^{T}<0 \text { and } \hat{v}_{i, j+1 / 2}^{B}+\hat{v}_{i, j+1 / 2}^{T}<0, \\ 0 & \text { otherwise, }\end{cases}
$$




$$
\hat{\boldsymbol{u}}_{i, j+1 / 2}= \begin{cases}\hat{\boldsymbol{u}}_{i, j+1 / 2}^{B} & \text { if } \hat{v}_{i, j+1 / 2}^{\mathrm{adv}}>0, \\ \hat{\boldsymbol{u}}_{i, j+1 / 2}^{T} & \text { if } \hat{v}_{i, j+1 / 2}^{\mathrm{adv}}<0, \\ \left(\hat{\boldsymbol{u}}_{i, j+1 / 2}^{B}+\hat{\boldsymbol{u}}_{i, j+1 / 2}^{T}\right) / 2 & \text { if } \hat{v}_{i, j+1 / 2}^{\mathrm{adv}}=0 .\end{cases}
$$

\subsection{Thickness of the interface}

Because of the numerical difficulty caused by the Dirac delta function and by the sharp change of $\rho$ and $v$ across the free surface, the Heaviside and Dirac delta functions are replaced by smoothed functions. The Heaviside function (see Sussman et al. [23]) is redefined as

$$
H(\phi)= \begin{cases}0 & \text { if } \phi<-\epsilon, \\ \frac{1}{2}\left[1+\frac{\phi}{\epsilon}+\frac{1}{\pi} \sin (\pi \phi / \epsilon)\right] & \text { if }|\phi| \leqslant \epsilon, \\ 1 & \text { if } \phi>\epsilon .\end{cases}
$$

Accordingly, the smoothed delta function is

$$
\delta(\phi)=\frac{\mathrm{d} H(\phi)}{\mathrm{d} \phi} .
$$

It is clear that the thickness of the interface is $2 \epsilon$ if the level set is a distance function.

In our numerical calculation, the parameter $\epsilon$ is chosen to be related to the mesh size

$$
\epsilon=\frac{\alpha}{2}(\Delta r+\Delta z)
$$

where $\alpha=2$ in this work. The thickness of the interface reduces as we refine the mesh. Nonetheless, for any numerically practical choices, the interface will necessarily have some smearing.

\subsection{Constraint on time step}

Since our time integration scheme is 2 nd-order explicit for the convection term and 1st-order explicit for viscosity, the constraint on time step $\Delta t$ is determined by the CFL condition, surface tension, viscosity, and total acceleration (Sussman et al. [21]), as in

$$
\Delta t<\min _{i, j}\left[\frac{\Delta r}{u}, \frac{\Delta z}{v}, \sqrt{\mathrm{We} \frac{\rho_{1}+\rho_{2}}{8 \pi}} h^{3 / 2}, \frac{\operatorname{Re}}{2} \frac{\rho^{n}}{\mu^{n}}\left(\frac{1}{\Delta r^{2}}+\frac{1}{\Delta z^{2}}\right)^{-1}, \sqrt{\frac{2 h}{|\boldsymbol{F}|}}\right],
$$

where $h=\min (\Delta r, \Delta z)$ and $\boldsymbol{F}$ is defined in 3.14.

\subsection{Formulation of FEM projection}

We let $\boldsymbol{u}^{*}$ be piecewise constant while $p^{n+1}$ and $\psi$ be piecewise bilinear. For the term $\int_{\Omega} \frac{\Delta t}{\rho\left(\phi^{n+1 / 2}\right)} \nabla p^{n+1} \cdot \nabla \psi \mathrm{d} \boldsymbol{x}$, we show how to derive the integral related to the weighting function $\psi_{i, j}$. As shown in Figure 6(a), the contribution to the $\psi_{i, j}$-related integral comes from the cell with corner points $A_{1}, A_{2}, A_{3}, A_{4}$ because the weighting function $\psi_{i, j}$ has the value $\hat{\psi}_{i, j}$ at node $(i, j)$ and vanishes at all other nodes. 
(a)

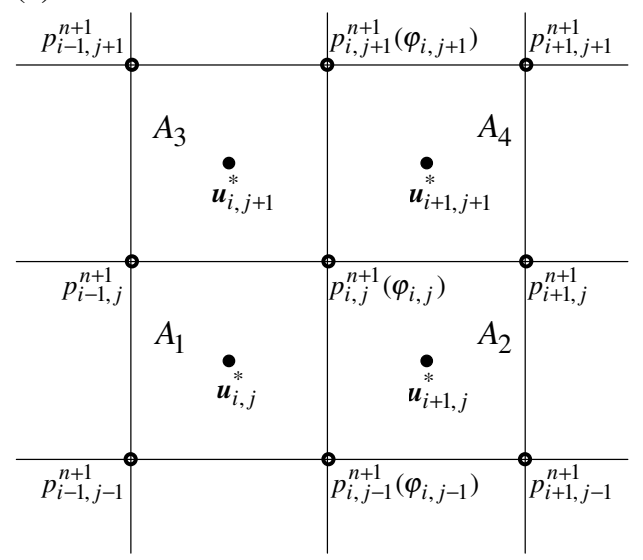

(b)

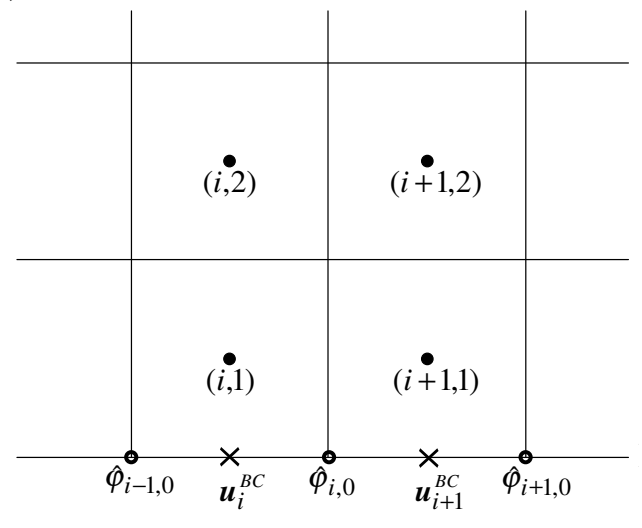

FIG. 6. Location of variables: (a) velocity $\boldsymbol{u}^{*}$, pressure $p_{i, j}^{n+1}$, and shape fcn. $\psi_{i, j}$; (b) velocity $\boldsymbol{u}^{*}$, pressure, and shape fcn.

The contribution from $A_{1}$ is

$$
\begin{aligned}
& \int_{A_{1}}\left(\frac{1}{\rho(\phi)} \nabla p^{n+1}\right) \cdot \nabla \psi_{i, j} \mathrm{~d} \boldsymbol{x}=\int_{A_{1}}\left(\frac{1}{\rho_{i, j}} \nabla p^{n+1}\right) \cdot \nabla \psi_{i, j} \mathrm{~d} \boldsymbol{x} \\
&=\frac{2 \pi}{\rho_{i, j}(\Delta r \Delta z)^{2}} \int_{z=-\Delta z}^{0} \int_{r=-\Delta r}^{0} \nabla\left[p_{i, j}^{n+1}(r+\Delta r)(z+\Delta z)-p_{i-1, j}^{n+1} r(z+\Delta z)\right. \\
&\left.-p_{i, j-1}^{n+1}(r+\Delta r) z+p_{i-1, j-1}^{n+1} r z\right] \cdot \nabla\left[\hat{\psi}_{i, j}(r+\Delta r)(z+\Delta z)\right]\left(r_{i, j}+r\right) \mathrm{d} r \mathrm{~d} z \\
&= \frac{2 \pi}{3 \rho_{i, j}} \hat{\psi}_{i, j}\left[p_{i, j}^{n+1}\left(\frac{\Delta z}{\Delta r} Q_{1} r_{i, j}-\frac{\Delta z}{2} Q_{3}\right)-p_{i-1, j}^{n+1}\left(\frac{\Delta z}{\Delta r} r_{i, j}-\frac{\Delta z}{2}\right) Q_{4}\right. \\
&\left.-p_{i, j-1}^{n+1}\left(\frac{\Delta r}{\Delta z} Q_{5} r_{i, j}+\frac{\Delta z}{4} Q_{2}\right)-p_{i-1, j-1}^{n+1}\left(\frac{\Delta z}{2 \Delta r} r_{i, j}-\frac{\Delta z}{4}\right) Q_{1}\right]
\end{aligned}
$$

where $r_{i, j}$ is the radius at the center of cell $(i, j)$ and

$$
\begin{array}{rlrl}
Q_{1} & =1+\frac{\Delta r^{2}}{\Delta z^{2}}, & Q_{2} & =1-\frac{\Delta r^{2}}{\Delta z^{2}}, \\
Q_{3} & =1+\frac{\Delta r^{2}}{2 \Delta z^{2}}, & Q_{4} & =1-\frac{\Delta r^{2}}{2 \Delta z^{2}}, \\
Q_{5} & =1-\frac{\Delta z^{2}}{2 \Delta r^{2}} . &
\end{array}
$$

Similarly, one can obtain the contribution to the $\psi_{i, j}$-related integral from $A_{2}, A_{3}$, and $A_{4}$.

For the term $\int_{\Omega} \boldsymbol{u}^{*} \cdot \nabla \psi \mathrm{d} \boldsymbol{x}$, we show all the contribution from the point $A_{1}$, which includes the $\psi_{i, j}, \psi_{i, j-1}, \psi_{i-1, j-1}$, and $\psi_{i-1, j}$-related quantities:

$$
\begin{aligned}
\int_{A_{1}} \boldsymbol{u}^{*} \cdot \nabla \psi \mathrm{d} \boldsymbol{x}= & \frac{2 \pi}{\Delta r \Delta z} \int_{z=-\Delta z}^{0} \int_{r=-\Delta r}^{0}\left\{\hat{\psi}_{i, j} \nabla[(r+\Delta r)(z+\Delta z)]-\hat{\psi}_{i, j-1} \nabla[(r+\Delta r) z]\right. \\
& \left.-\hat{\psi}_{i-1, j} \nabla[r(z+\Delta z)]+\hat{\psi}_{i-1, j-1} \nabla(r z)\right\} \cdot \boldsymbol{u}_{i, j}^{*}\left(r_{i, j}+r\right) \mathrm{d} r \mathrm{~d} z
\end{aligned}
$$




$$
\begin{aligned}
= & \pi\left\{\hat{\psi}_{i, j}\left[\left(\Delta r v_{i, j}^{*}+\Delta z u_{i, j}^{*}\right) r_{i, j}-\frac{\Delta r}{2}\left(\Delta z u_{i, j}^{*}+\frac{2 \Delta r}{3} v_{i, j}^{*}\right)\right]\right. \\
& -\hat{\psi}_{i, j-1}\left[\left(\Delta r v_{i, j}^{*}-\Delta z u_{i, j}^{*}\right) r_{i, j}+\frac{\Delta r}{2}\left(\Delta z u_{i, j}^{*}-\frac{2 \Delta r}{3} v_{i, j}^{*}\right)\right] \\
& +\hat{\psi}_{i-1, j}\left[\left(\Delta r v_{i, j}^{*}-\Delta z u_{i, j}^{*}\right) r_{i, j}+\frac{\Delta r}{2}\left(\Delta z u_{i, j}^{*}-\frac{4 \Delta r}{3} v_{i, j}^{*}\right)\right] \\
& \left.-\hat{\psi}_{i-1, j-1}\left[\left(\Delta r v_{i, j}^{*}+\Delta z u_{i, j}^{*}\right) r_{i, j}-\frac{\Delta r}{2}\left(\Delta z u_{i, j}^{*}+\frac{4 \Delta r}{3} v_{i, j}^{*}\right)\right]\right\} .
\end{aligned}
$$

For the boundary integral $\int_{\Gamma_{1}} \psi \boldsymbol{u}^{B C} \cdot \boldsymbol{n} \mathrm{d} S$, we show the formula for the case that $\Gamma_{1}$ is a horizontal line at the bottom of the solution domain (see Figure $6(\mathrm{~b})$ ). We also assume that $\boldsymbol{u}^{B C}$ is given at the middle point of each cell edge and is piecewise constant. The integral for the bottom edge of the cell $(i, 1)$ is thus given by

$$
\begin{aligned}
\int_{\text {bottom of }(i, 1)} \psi \boldsymbol{u}^{B C} \cdot \boldsymbol{n} \mathrm{d} S & =\int_{-\Delta r}^{0} \frac{2 \pi}{\Delta r}\left[\hat{\psi}_{i, 0}(r+\Delta r)-\hat{\psi}_{i-1,0} r\right]\left(-v_{i}^{B C}\right)\left(r_{i, 0}+r\right) \mathrm{d} r \\
& =-\pi \Delta r\left[\hat{\psi}_{i, 0}\left(r_{i, 0}-\frac{\Delta r}{3}\right)+\hat{\psi}_{i-1,0}\left(r_{i, 0}-\frac{2 \Delta r}{3}\right)\right] v_{i}^{B C} .
\end{aligned}
$$

\section{Numerical results}

\subsection{Convergence study}

For a convergence check of our code for ink jet simulation, we considered a typical nozzle as in Figure 1. The diameter is 26 microns at the opening and 65 microns at the bottom. The length of the nozzle opening part, where the diameter is 26 microns, is 20.8 microns. The slant part is 59.8 microns and the bottom part is 7.8 microns.

The inflow pressure is given by an equivalent circuit which simulates the effect of the ink cartridge, supply channel, vibration plate, PZT actuator, applied voltage, and the ink inside the channel and cartridge. We assumed that the input voltage is given by Figure 3 The corresponding inflow pressure is as shown in Figure 4 . The outflow pressure at the top of the solution domain is set to zero.

The solution domain was chosen to be $\{(r, z) \mid 0 \leqslant r \leqslant 39 \mu \mathrm{m}, 0 \leqslant z \leqslant 312 \mu \mathrm{m}\}$. Since the slant part of the nozzle wall is not parallel to the coordinate axes, we applied the "method of obstacle cells" (see Griebel et al. [12]) in discretizing the nozzle geometry using homogeneous rectangular cells. As a result the nozzle wall has a staircase pattern (see Fig. 7). A better approximation would result from using a body-fitted quadrilateral mesh. In [25], the projection method is extended to an arbitrary general quadrilateral mesh. Those results indicate that the difference between using a staircase representation vs. a body-fitted mesh, while important for small-scale boundary effects, is not significant in terms of computing the pinch-off time, bubble velocity, and satellite formation.

The contact angle was assumed to be $90^{\circ}$ all the time and the initial meniscus is assumed to be flat and 2.6 microns lower than the nozzle opening.

For the purpose of normalization, we chose the nozzle opening diameter (26 microns) to be the length scale and $6 \mathrm{~m} / \mathrm{sec}$ to be the velocity scale. The normalized solution domain is hence 


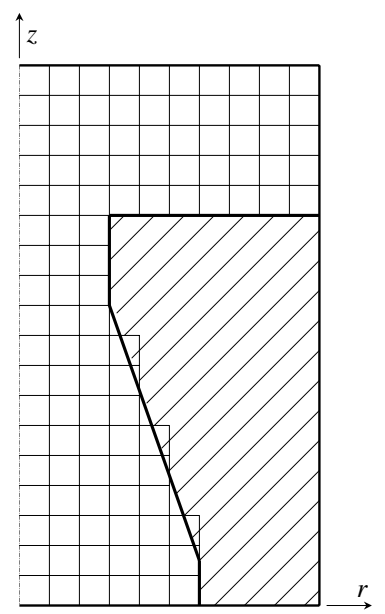

FIG. 7. The rectangular mesh.

$\{(r, z) \mid 0 \leqslant r \leqslant 1.5,0 \leqslant z \leqslant 12\}$. Since the density, viscosity, and surface tension of ink are approximately

$$
\rho_{1}=1070 \mathrm{Kg} / \mathrm{m}^{3}, \quad \mu_{1}=3.34 \times 10^{-3} \mathrm{Kg} / \mathrm{m} \cdot \mathrm{sec}, \quad \sigma=0.032 \mathrm{Kg} / \mathrm{sec}^{2},
$$

we have the following nondimensional parameters:

$$
\operatorname{Re}=50 ., \quad \mathrm{We}=31.3 .
$$

The density and viscosity of air are

$$
\rho_{2}=1.225 \mathrm{Kg} / \mathrm{m}^{3}, \quad \mu_{2}=1.77625 \times 10^{-3} \mathrm{Kg} / \mathrm{m} \cdot \mathrm{sec} .
$$

To check the convergence of our code, we list in Tables 1, 2, and 3 the time of droplet pinch off, droplet head velocity, and droplet volume obtained from our code using various meshes.

It is seen that the $25 \times 200$ mesh does not conserve mass well. This is because the widest part of the ink droplet is only 7 to 8 cells wide. On such coarse meshes, level set methods incur substantial mass loss. While remedies have been proposed, they typically maintain global mass conservation but suffer from local mass loss. The most effective remedy is a careful attention to re-initialization issues, higher order schemes, and sufficient grid resolutions.

TABLE 1

The time to pinch off from various meshes

\begin{tabular}{||l||c|c|c|c||}
\hline \hline Mesh number & $25 \times 200$ & $50 \times 400$ & $75 \times 600$ & $100 \times 800$ \\
\hline Time to pinch off & 8.0944 & 8.1808 & 8.1755 & 8.1738 \\
\hline \hline
\end{tabular}


TABLE 2

Droplet head velocities from various meshes

\begin{tabular}{||l||c|c|c|c||}
\hline \hline Mesh number & $25 \times 200$ & $50 \times 400$ & $75 \times 600$ & $100 \times 800$ \\
\hline$t=3.20$ & 1.494 & 1.411 & 1.471 & 1.496 \\
\hline$t=4.48$ & 1.245 & 1.188 & 1.219 & 1.246 \\
\hline$t=5.76$ & 1.047 & 1.012 & 1.065 & 1.093 \\
\hline$t=6.72$ & 0.972 & 0.932 & 0.990 & 1.023 \\
\hline \hline
\end{tabular}

TABle 3

Droplet volumes from various meshes

\begin{tabular}{||l||c|c|c|c||}
\hline \hline Mesh number & $25 \times 200$ & $50 \times 400$ & $75 \times 600$ & $100 \times 800$ \\
\hline$t=8.64$ & 0.5768 & 0.7318 & 0.7408 & 0.7440 \\
\hline$t=8.96$ & 0.5659 & 0.7279 & 0.7389 & 0.7429 \\
\hline$t=9.28$ & 0.5548 & 0.7236 & 0.7369 & 0.7417 \\
\hline$t=9.60$ & 0.5416 & 0.7189 & 0.7347 & 0.7405 \\
\hline$t=9.96$ & 0.5281 & 0.7140 & 0.7325 & 0.7396 \\
\hline \hline
\end{tabular}

\subsection{Effect of contact model}

Our results indicate that the choice of contact model has an effect on the results. For the first droplet, the dependence on the particular contact model is not that significant. We simulated the continuous droplet ejection by repeating the voltage pattern in Fig. 3 at a frequency of $14.4 \mathrm{KHz}$. Results with
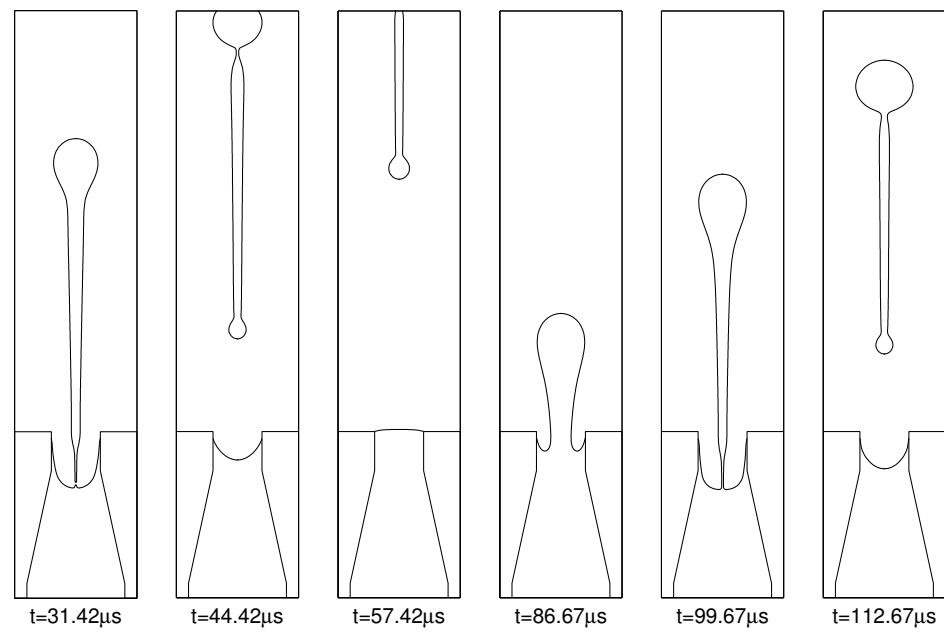

FIG. 8. The continuous droplet ejection with the use of the contact model. 

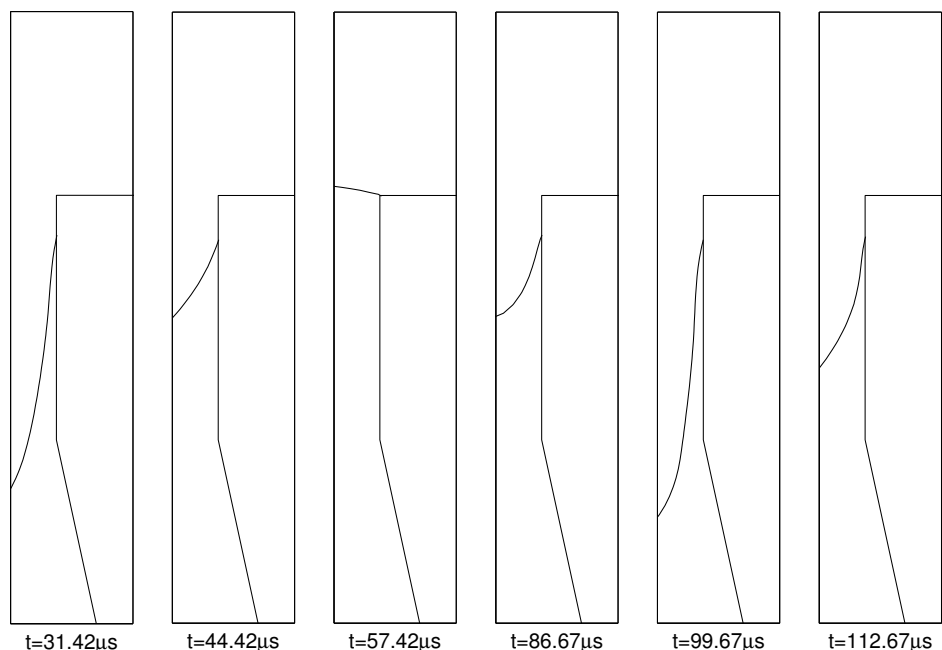

FIG. 9. A close up view at the triple point (with the contact model).
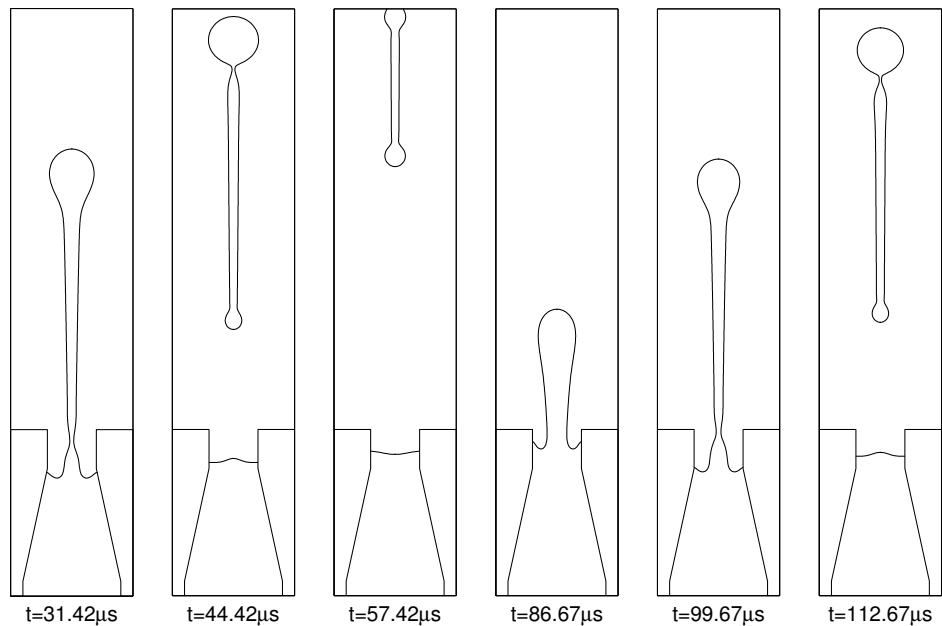

FIG. 10. The continuous droplet ejection by assuming that the contact angle is always $90^{\circ}$.

the contact model and results assuming a constant contact angle of $90^{\circ}$ are plotted in Figs. 8 and 10 . Close up views at the triple point are shown in Figs. 9 and 11 .

We see from Figs. 8 and 9 that the center of the meniscus retreats deep into the nozzle when the first droplet pinches off at $t=31.42 \mu \mathrm{s}$. The ink droplet continues moving upward and breaks into one major droplet and one or more satellites. At the same time, the capillary force of the meniscus pulls the ink in the nozzle upward so that the ink flows from the cartridge into the nozzle. The meniscus returns to the nozzle opening and recovers to almost a straight line before the nozzle is ready for the next ejection $(t=57.42 \mu \mathrm{s})$. Thus the second droplet as shown in Fig. 8 is slightly bigger 

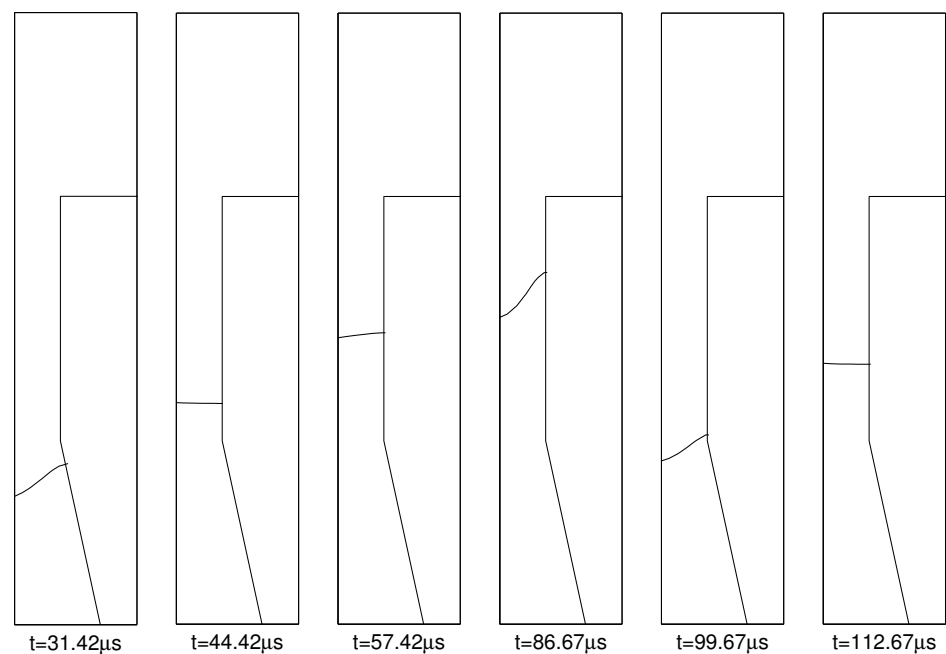

FIG. 11. A close up view at the triple point $\left(\right.$ contact angle $\left.=90^{\circ}\right)$.

than the first one. This transient phenomenon is consistent with our experimental observation. If the simulation is continued, the third, fourth, and later droplets are basically as big as the second droplet.

If the contact angle is assumed to be $90^{\circ}$ all the time, as is the case in Fig. 10, the capillary force is not big enough at the pinch off (slightly later than $t=31.42 \mu \mathrm{s}$ ) of the first droplet. Hence the nozzle is not correctly refilled before the start of the second ejection. Subsequent droplets are smaller than the initial droplet, which is contrary to experimental observations.

\subsection{Experimental verification}

An experimental result is plotted in Fig. 13. The nozzle used in the experiment is 25 microns in diameter at the opening and 49.5 microns at the bottom. The length of the nozzle opening part, where the diameter is 25 microns, is 25 microns. The slant part is 55 microns long and the bottom part is 7.5 microns. The nozzle is not shown in Fig. 13 but the position of nozzle exit is at the upper edge of each of the six images. The density, viscosity, and surface tension of Epson's dye-based ink are

$$
\rho_{1}=1070 \mathrm{Kg} / \mathrm{m}^{3}, \quad \mu_{1}=3.7 \times 10^{-3} \mathrm{Kg} / \mathrm{m} \cdot \mathrm{sec}, \quad \sigma=0.032 \mathrm{Kg} / \mathrm{sec}^{2} .
$$

The dynamic voltage used in the experiment is plotted in Fig. 12, where the peak voltages are \pm 10.96 volts. Simulation results are shown in Fig. 14

The average droplet size obtained from the experiment is 12.928 pico liters. Simulation of this ink jet using a $64 \times 672$ grid predicts 11.659 pico liters. Fig. 13 shows that the ink droplet leaves the nozzle at a time between $30 \mu$ s and $35 \mu$ s. Since the pinch off from the meniscus usually happens somewhere inside the nozzle, we cannot see it in the experiment. Our simulation predicts that the pinch off occurs at $t=30.80 \mu \mathrm{s}$ and that the droplet tail leaves the nozzle at $t=31.50 \mu \mathrm{s}$, in good agreement with experiments. 
INK JET SIMULATION

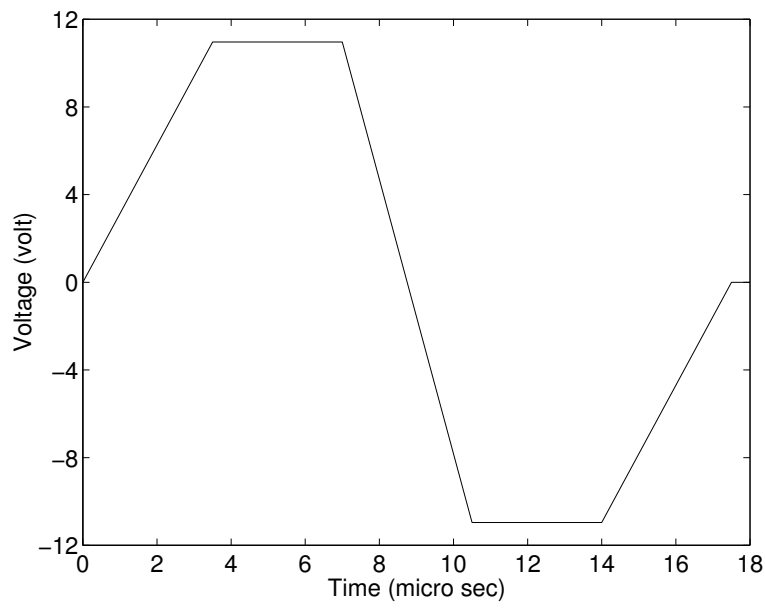

FIG. 12. The driving voltage used in experiment.
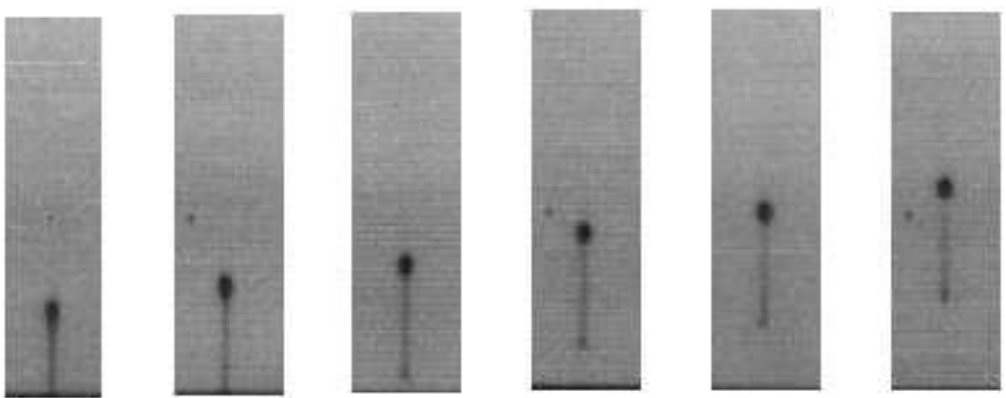

FIG. 13. Shape of the ink droplet at $t=25,30,35,40,45,50 \mu s$.
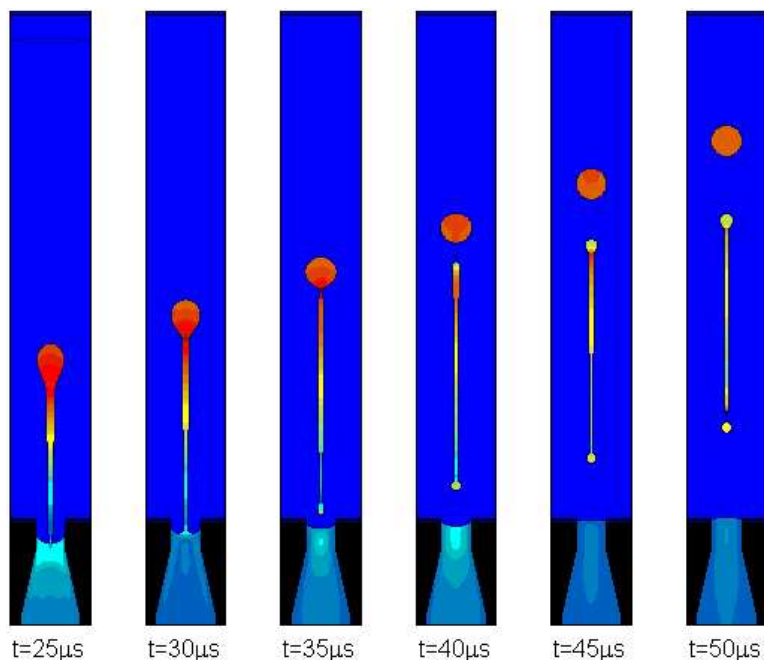

FIG. 14. Ejection of an ink droplet from simulation. 


\subsection{Effect of parameters}

The effects of some design parameters can be seen using the simulation code. Here we only illustrate two of them. The relation between the ink droplet volume and the peak voltage is plotted in Fig. 15 . Although the two-phase flow problem is highly nonlinear, the curve in Fig. 15 is essentially linear. The droplet velocity increases and the "time to droplet pinch off" becomes longer as the peak voltage increases. As a result, the ejected droplet gets longer and, due to the capillary instability, tends to develop into more satellites. Hence increasing the driving voltage is not a solution for bigger ink droplets. The magnitude of surface tension coefficient does not influence the droplet size as much as the peak voltage, but it affects the time of pinch off. Plotted in Fig. 16 is the relation between pinch off time and surface tension coefficient, showing that a larger surface tension causes earlier pinch off.

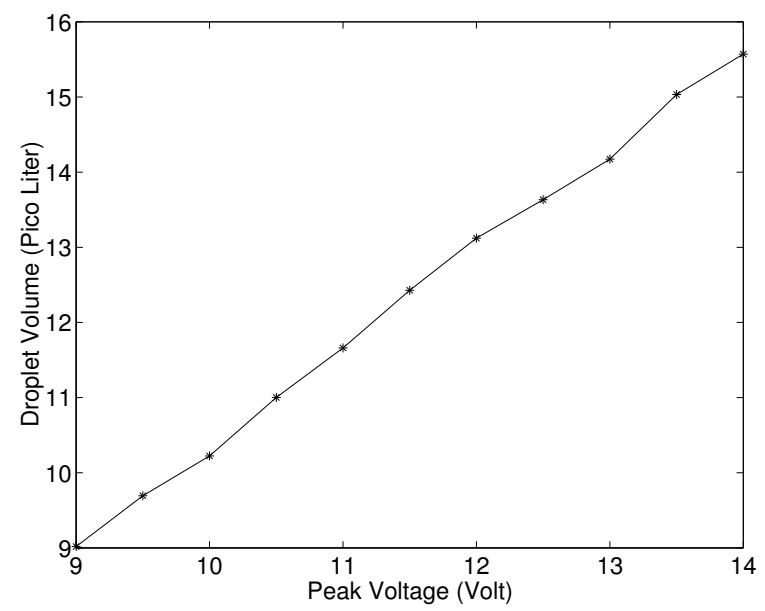

FIG. 15. The relation between the droplet volume and the peak value of the driving voltage.

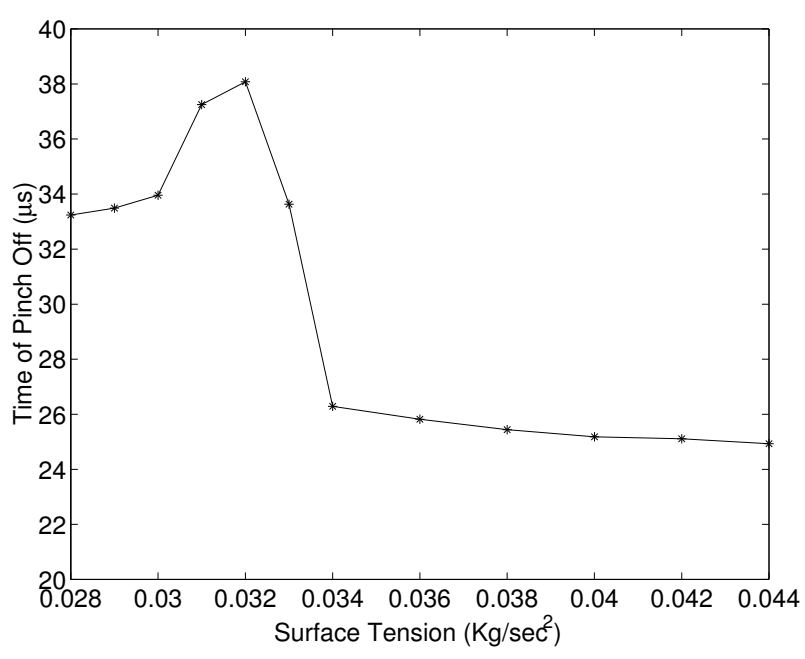

FIG. 16. The relation between the time of pinch off and the surface tension. 


\section{Summary}

We have developed an axisymmetric second-order coupled level set projection method with contact model to model flow in an ink jet printhead, performed numerical simulations to check internal consistency, and compared results with experimental data. In the future, we plan a closer examination of wall forces, thin structures and temperature terms.

\section{Acknowledgements}

We would like to thank Dr. Ann Almgren, Dr. John Bell, and Professor David Chopp for many valuable conversations.

\section{REFERENCES}

1. Aleinov, I. D., Puckett, E. G., \& Sussman, M. Formation of droplets in microscale jetting devices. Proc. ASME FEDSM'99 (San Francisco, CA 1999).

2. Almgren, A. S., Bell, J. B., \& Crutchfield, W. Y. Approximate projection methods: Part I. Inviscid analysis. SIAM J. Sci. Comput. 22 (2000), 1139-1159. Zbl 0995.76059 MR 2001f:76060

3. Almgren, A. S., Bell, J. B., \& SzymczaK, W. G. A numerical method for the incompressible Navier-Stokes equations based on an approximate projection. SIAM J. Sci. Comput. 17 (1996), 358-369. Zbl 0845.76055 MR 96j:76104

4. Bell, J. B., Colella, P., \& Glaz, H. M. A second-order projection method for the incompressible Navier-Stokes equations. J. Comput. Phys. 85 (1989), 257-283. Zbl 0681.76030 MR 90i:76002

5. Bell, J. B. \& MARCUS, D. L. A second-order projection method for variable-density flows. J. Comput. Phys. 101 (1992), 334-348. Zbl 0759.76045

6. Bertozzi, A. The mathematics of moving contact lines in thin liquid films. Notices Amer. Math. Soc. 45 (1998), 689-697. Zbl 0917.35100 MR 99h:76032

7. Chang, Y. C., Hou, T. Y., Merriman, B., \& Osher, S. A level set formulation of Eulerian interface capturing methods for incompressible fluid flows. J. Comp. Phys. 124 (1996), 449-464. Z Zbl 0847.76048 MR 97a:76087

8. Chopp, D. L. Computing minimal surfaces via level set curvature flow. J. Comput. Phys. 106 (1993), 77-91. Zbl 0786.65015 MR 94f:53007

9. Chorin, A. J. Numerical solution of the Navier-Stokes equations. Math. Comput. 22 (1968), 745-762. Zbl 0198.50103 MR 39\#3723

10. Colella, P. A direct Eulerian MUSCL scheme for gas dynamics. SIAM J. Sci. Statist. Comput. 6 (1985), 104-117. Zbl 0562.76072 MR 86g:65156

11. Colella, P. Multidimensional upwind methods for hyperbolic conservation laws. SIAM J. Sci. Statist. Comput. 87 (1990), 171-200. Zbl 0694.65041 MR 91c:76087

12. Griebel, M., Dornseifer, T., \& NeunhoefFer T. Numerical Simulation in Fluid Dynamics: A Practical Introduction. SIAM, (1998), 45-49. Zbl 0945.76001 MR 99a:76078

13. Osher, S. \& Sethian, J. A. Fronts propagating with curvature-dependent speed: algorithms based on Hamilton-Jacobi formulations. J. Comput. Phys. 79 (1988), 12-49. Zbl 0659.65132 MR 89h:80012

14. Puckett, E. G., Almgren, A. S., Bell, J. B., Marcus, D. L., \& Rider, W. J. A high-order projection method for tracking fluid interfaces in variable density incompressible flows. J. Comput. Phys. 130 (1997), 269-282. Zbl 0872.76065

15. SAKAI, S. Dynamics of piezoelectric inkjet printing systems. Proc. IS\&T NIP 16 (2000), 15-20.

16. Sethinan, J. A. An analysis of flame propagation. Ph.D. Dissertation, Department of Mathematics, University of California, Berkeley, CA (1982). 
17. Sethinan, J. A. Curvature and the evolution of fronts. Comm. Math. Phys. 101 (1985), 487-499. Zbl 0619.76087

18. Sethina, J. A. Numerical methods for propagating fronts. Variational Methods for Free Surface Interfaces, P. Concus \& R. Finn (eds.), Springer, NY (1987), 155-164. Zbl 0618.65128

19. Sethian, J. A. Level Set Methods and Fast Marching Methods. 2nd ed., Cambridge Univ. Press (1999). Zbl 0973.76003

20. Sou, A., SASAI, K., \& NAKAJIMA, T. Interface tracking simulation of ink jet formation by electrostatic force. Proc. ASME FEDSM'01 (New Orleans, 2001), 505-510.

21. Sussman, M., Almgren, A. S., Bell, J. B., Colella, P., Howell, L. H., \& Welcome, M. L. An adaptive level set approach for incompressible two-phase flow. J. Comput. Phys. 148 (1999), 81-124. Zbl 0930.76068 MR 99m:76098

22. Sussman, M. \& SMEReKa, P. Axisymmetric free boundary problems. J. Fluid Mech. 341 (1997), 269294. Zbl 0892.76090 MR 98d:76039

23. Sussman, M., Smereka, P. \& Osher, S. J. A level set approach for computing solutions to incompressible two-phase flow. J. Comput. Phys. 114 (1994), 146-159. Zbl 0808.76077

24. UnVERd, S. O. \& Tryggvason, G. A front-tracking method for viscous, incompressible, multi-fluid flows. J. Comput. Phys. 100 (1992), 25-37. Zbl 0758.76047

25. Yu, J.-D., Sakai, S., \& Sethian, J. A. A general quadrilateral mesh projection method for two-phase flow in body-fitted geometries. To be submitted (2003).

26. Zhu, J. \& Sethian, J. A. Projection methods coupled to level set interface techniques. J. Comput. Phys. 102 (1992), 128-138. Zbl 0751.76050 